\title{
ANÁLISE DAS MUDANÇAS DO USO AGRÍCOLA DA TERRAA PARTIR DE DADOS DE SENSORIAMENTO REMOTO MULTITEMPORAL NO MUNICÍPIO DE LUIS EDUARDO MAGALHÃES (BA - BRASIL)
}

\section{Agricultural land use changes analysis from multi-temporal remote sensing data in the municipality of Luis Eduardo Magalhães (Bahia - Brazil)}

Aline Brignol Menke Universidade de Brasília Brasília/DF - Brasil alinemenke@gmail.com

Osmar Abílio de Carvalho Junior Professor Dr. da Universidade de Brasília Brasília/DF - Brasil osmar.junior@pq.cnpq.br

Roberto Arnaldo Trancoso Gomes Professor Dr. adjunto da Universidade de Brasília. Brasília/DF - Brasil robertogomes@unb.br

Éder de Souza Martins Dr. e Pesquisador A da EMBRAPA Cerrados Brasília/DF - Brasil eder@cpac.embrapa.br

Sandro Nunes de Oliveira Universidade de Brasília Brasília/DF - Brasil sandronunes@unb.br

Artigo recebido para publicação em 27/08/2009 e aceito para publicação em 19/11/2009

RESUMO: O presente trabalho tem como objetivo quantificar o uso da Terra e suas mudanças no município de Luis Eduardo Magalhães usando dados de sensoriamento remoto. A região teve uma grande expansão da agricultura e do agronegócio desde a década de 1980, devido às condições ambientais favoráveis com terras planas e estação chuvosa bem definida. As principais culturas são soja, algodão, milho e café, mas o solo do Cerrado também produz frutas, feijão e arroz, além de pastagens. O método de detecção de mudança de pós-classificação foi utilizado para determinar as diferenças entre as imagens durante as últimas duas décadas. Esta metodologia requer a comparação de imagens classificadas independentemente, minimizando os problemas de normalização da atmosfera, diferenças de sensor e condições ambientais (fenologia, ciclos precipitação e umidade do solo). Imagens ALOS e Landsat foram 
Análise das mudanças do uso agrícola da terra a partir de dados de sensoriamento remoto multitemporal no Município de Luis Eduardo Magalhães (BA - Brasil)

Aline Brignol Menke, Osmar Abílio de Carvalho Junior, Roberto Arnaldo Trancoso Gomes, Éder de Souza Martins, Sandro Nunes de Oliveira

utilizadas para elaborar mapas a partir da classificação da cobertura e uso da Terra entre 1987 e 2008. O método utilizado para classificar cada imagem foi a interpretação visual. Durante os últimos 20 anos, os agricultores converteram cerca de 40\% das áreas de cerrado em áreas de agropecuária. As mudanças na cobertura e uso da Terra do município de Luis Eduardo Magalhães refletem os impactos das decisões tomadas na política agrícola e econômica.

Palavras-chave: Análise Multitemporal. Detecção de Mudança. Pós-classificação. Agronegócio. Uso da Terra.

ABSTRACT: The present work aims to quantify land use and land cover changes in Luis Eduardo Magalhães municipality using remote sensing data. The region presented a large expansion of the agriculture and agribusiness since the 1980s due to the environmental conditions favorable with flat lands and a well-defined rainy season. Main crops are soybean, cotton, maize and coffee, but the Cerrado soil also produces fruits, beans and rice, besides pastures. The post-classification change detection method was used to determine differences between images during the last two decades. This methodology requires the comparison of independently classified images, thereby minimizing problems of normalization for atmospheric, sensor differences and landscape conditions (phenology, precipitation cycles and soil moisture). ALOS and Landsat images were used to produce land cover classification maps between 1987 and 2008. The method used to classify each image was visual interpretation. During the last 20 years, farmers converted approximately $40 \%$ of savanna into farmlands. Land cover changes in the Luis Eduardo Magalhães reflect the impacts of management decisions made in agricultural and economic policy.

Keywords: Multitemporal Analysis; Change Detection; Post-classification; Agribusiness; Land Use.

\section{INTRODUÇÃO}

Estima-se que a taxa média de crescimento da área agrícola no Brasil será de 4,7\% ao ano na próxima década, uma das maiores do mundo (IICA, 2007). Grande parte deste crescimento ocorrerá no bioma Cerrado que desde a década de 70 tornou-se o foco das novas expansões agropecuárias, sendo considerada a última grande fronteira a ser explorada do globo (BORLAUG, 2002). Aproximadamente 40\% dos 204 milhões de hectares de Cerrado já foram convertidos em pastagens cultivadas, áreas agrícolas e outros tipos de uso (SANO et al., 2009, 2008). A viabilidade de ocupação e consolidação econômica do Cerrado brasileiro foram provenientes dos seguintes fatores: (a) disponibilidade de terras com relativa proximidade dos centros econômicos do país (regiões Sul e Sudeste); (b) competitividade nacional no mercado externo, principalmente na produção de grãos; (c) fortalecimento do capital financeiro privado com a reestruturação in- terna da produção e o estabelecimento do agronegócio; e (d) inovação tecnológica proveniente de uma política de ciência e tecnologia coordenada, principalmente, pela EMBRAPA(FONSECA et al., 2004; SICSÚ \& LIMA, 2000). Desta forma, observa-se um aumento da produção tanto devido ao crescimento da área plantada, como também pela maior eficiência e produtividade agrícola oriundas do aprimoramento tecnológico.

Dentro deste cenário, o Cerrado do Oeste da Bahia é a região com maior expansão agrícola no estado nas últimas duas décadas (IBGE, 2002). Os solos desenvolvidos sobre as regiões de Chapadas apresentam uma textura arenosa e média, excessivamente drenados, planos e favoráveis ao desenvolvimento da agricultura intensiva e mecanizada (CUNHA et al., 2001). O clima é classificado como subúmido e úmido, com precipitação entre 1200 e 1600 mm, caracterizada por uma estação de chuva e outra de seca (SEI, 1997). A deficiência de chuva para a agricultura é suprida pela implantação de projetos de irrigação que utilizam as 
águas dos tributários do São Francisco, que se caracterizam por serem perenes e com boa vazão, ou do aqüífero Urucuia por meio de poços tubulares profundos. A conjunção de terras propícias a mecanização e a existência de água tanto, para plantio de sequeiro ou com irrigação, estabelecem condições ideais para a implantação do agronegócio voltado para o mercado internacional de soja e de algodão. As principais culturas plantadas no Oeste da Bahia são: soja, algodão, milho e café, sendo também representativas a produção de frutas, feijão, arroz e gramíneas para a formação de pastagens. A soja é a principal cultura da região, ocupando aproximadamente $60 \%$ da área cultivada.

Entretanto, o problema relacionado a essa rápida expansão é o comprometimento da qualidade ambiental. Conforme Cunha et al. (2001), o sistema de manejo convencional provoca mudanças nas propriedades edáficas dos solos, que acarreta perdas de produção já no terceiro ano de uso agrícola. O tempo de pousio de três anos não tem se mostrado suficiente para recompor as características edáficas na vegeta- ção de cerrado nativo, principalmente, considerando a matéria orgânica e água disponível. Além disso, o crescente uso da água para atender à demanda de projetos de irrigação causa conflitos para outorgas de água (GASPAR, 2006). Desta forma, torna-se necessário um sistema de controle para gestão e monitoramento do uso dos recursos naturais que considere a alta dinâmica agrícola da região. Neste propósito podem ser utilizadas técnicas de sensoriamento remoto que apresentam como vantagem recobrir grandes extensões de áreas com custos reduzidos (ROCHA \& ROSA, 2007).

O objetivo desse trabalho é avaliar as modificações espaciais de uso e ocupação transcorridas nas últimas duas décadas (do final da década de 80 até 2008), utilizando técnicas de sensoriamento remoto, onde hoje se localiza o município de Luis Eduardo Magalhães (LEM) (FIG. 1). Este município emancipou-se em 2001 do município de Barreiras e é denominado de “capital do agronegócio”. Os investimentos na área do município se acentuaram pela iniciativa privada e pelo governo baiano, a partir da década de 90,

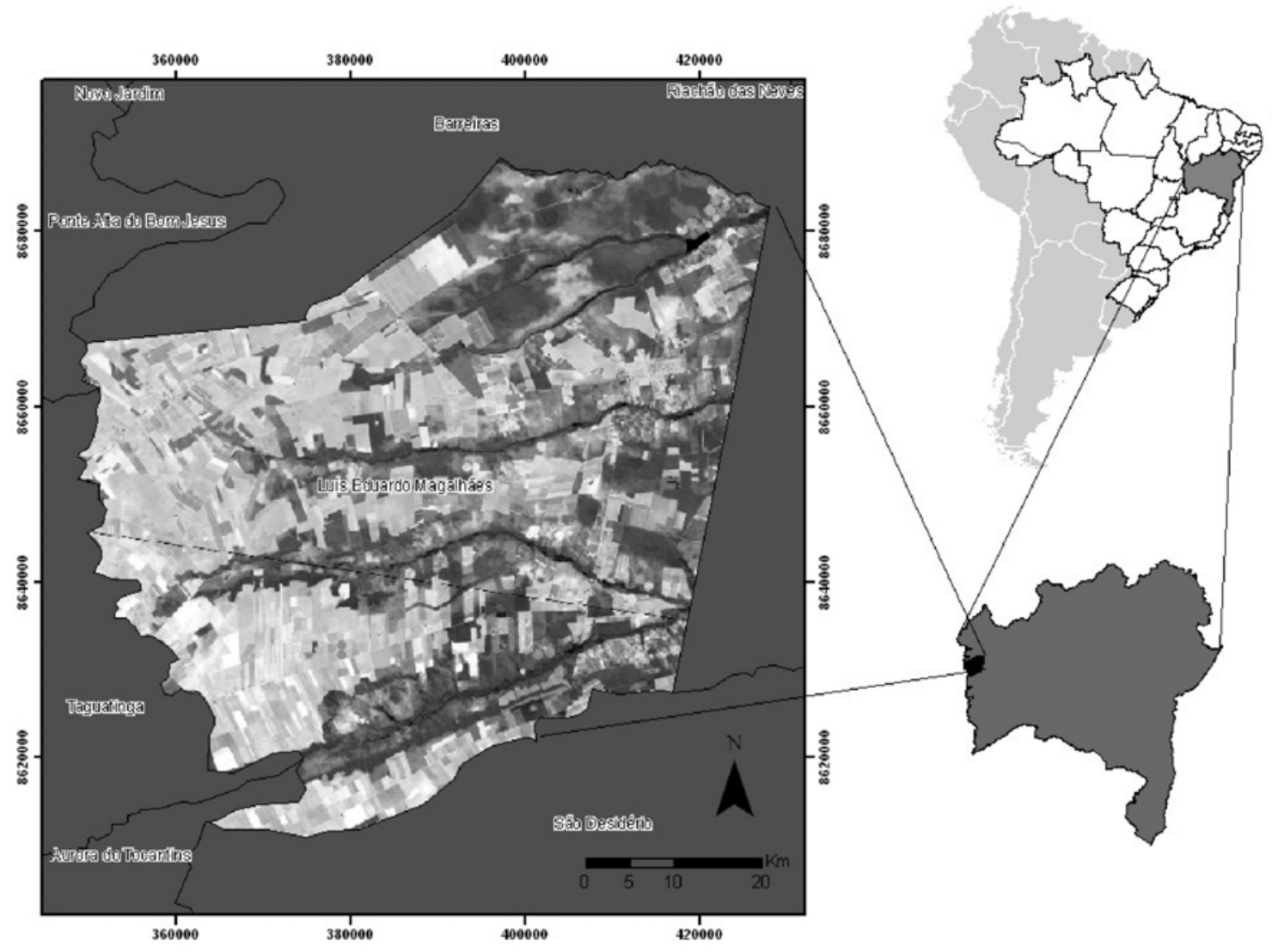

FIGURA 1: Mapa de localização da área de estudo. 
Análise das mudanças do uso agrícola da terra a partir de dados de sensoriamento remoto multitemporal no Município de Luis Eduardo Magalhães (BA - Brasil)

Aline Brignol Menke, Osmar Abílio de Carvalho Junior, Roberto Arnaldo Trancoso Gomes, Éder de Souza Martins, Sandro Nunes de Oliveira

gerando novas estruturas agroindustriais e urbanas. Atualmente, o município possui uma população de 44.265 habitantes, além de ser responsável por 1,3\% da produção nacional (IBGE, 2007).

\section{MATERIAIS E MÉTODOS}

\subsection{Imagens dos sensores ALOS e Landsat}

No presente trabalho foram utilizadas imagens de alta resolução do sensor PRISM/ALOS referente ao ano de 2008 e dos sensores TM e ETM/Landsat para os anos de 1987, 1991, 1994, 1998, 2001, 2004, 2005 e 2007 para remontar a série temporal.

O satélite ALOS (Advanced Land Observing Satellite) da Japan Aerospace Exploration Agency (JAXA) foi lançado em 2006, com o objetivo de promover o avanço na tecnologia de observação da superfície terrestre e contribuir para o estudo cartográfico, observação regional, monitoramento de desastres e pesquisa em recursos naturais. Este sensor possui uma órbita circular heliossíncrona, altitude de $692 \mathrm{~km}$, e resolução temporal de 46 dias (ciclo). Este satélite é constituído por três sensores: (a) Panchromatic Remote Sensing Instruments for Stereo Mapping (PRISM), Advanced Visible and Near Infrared Radiometer type 2 (AVNIR-2) e Phased Array type L-band Synthetic Aperture Radar (PALSAR) (IGARASHI, 2001).

No presente trabalho são utilizadas as imagens pancromáticas de 8 bits do sensor PRISM com resolução espacial de 2,5 m. Composto por um conjunto de 3 sistemas de aquisição de imagem, denominado Triplet, o PRISM obtém de forma simultânea imagens com visadas nadir, inclinada para frente e inclinada para trás, o que torna possível a aquisição de imagens estereoscópicas ao longo da trajetória e a construção do modelo digital de terreno. A largura da faixa de imageamento é de $35 \mathrm{~km}$ no modo de observação estéreo e de $70 \mathrm{~km}$ em observação nadir (TADONO et al., 2004). Estudos demonstram que o alinhamento relativo dos chips CCD dentro das câmaras não são perfeitos necessitando de uma calibração (JAXA, 2006). Modelos de auto-calibração tem sido propostos com o objetivo de aumentar a acurácia espacial dos dados baseados nas informações da JAXA sobre o sensor CCD (KOCAMAN \& GRUEN, 2008; JAXA,
2006; WESER et al., 2008). No presente trabalho foram adquiridas imagens referentes ao produto $1 \mathrm{~B} 2$ das imagens PRISM, que são submetidas à calibração radiométrica e geométrica, com os pixels alinhados com a grade da projeção UTM. O erro médio quadrático da precisão geométrica absoluta para a visão nadir é de $8 \mathrm{~m}$ (cross track) e $9 \mathrm{~m}$ (along track), enquanto que a precisão relativa é de $4 \mathrm{~m}$ (cross trtack) e $3 \mathrm{~m}$ (along track) (JAXA, 2007).

As imagens dos sensores Landsat Thematic Mapper (TM) e Enhanced Thematic Mapper Plus $(\mathrm{ETM}+)$ utilizadas são referentes às seis bandas da faixa do visível e infravermelho com resolução de 30 metros. Estas imagens foram co-registradas com as imagens ALOS para obter uma precisa sobreposição.

\subsection{Detecção de mudança pelo método de pós- classificação}

Os métodos de detecção de mudança buscam identificar alterações no estado de um objeto, ou nas feições da superfície terrestre, entre imagens de diferentes datas (SINGH, 1989). No presente trabalho, o método adotado para a detecção de mudança foi a pós-classificação. Neste método as imagens são previamente classificadas, individualmente, de forma manual ou por métodos computacionais e depois são comparadas extraindo e quantificando as áreas de mudança, podendo utilizar a técnica de tabulação cruzada (HOWARTH \& WICKWARE, 1981; MAS, 1999; MUNYATI, 2000 e DE BRUIN, 2000). Esta técnica de detecção de mudança apresenta as seguintes vantagens: (a) fácil atualização ao longo do tempo favorecendo o monitoramento; (b) permite compensar as variações provenientes das condições atmosféricas, mudanças fenológicas e umidade de solo, devido à independência na confecção do mapa temático; e (c) permite integrar e comparar imagens de sensores com diferentes resoluções espaciais, espectrais, temporais e radiométricas (COPPIN et al., 2004; NARUMALANI et al., 2004). Como desvantagens têm-se os seguintes fatores: (a) não é completamente automático, tornando-se um processo mais lento; e (b) a precisão da detecção das mudanças depende da acurácia da classificação em cada tempo, o que pode facilitar a propagação de erros. 
No presente trabalho foi realizada inicialmente a interpretação visual em tela das imagens do sensor PRISM referente ao ano de 2008. Com base nesta primeira classificação, utilizando a imagem de maior resolução espacial, foi feita uma retroanálise para os anos anteriores, também por interpretação visual, a partir de imagens do sensor Landsat. Cabe salientar que muitas das diferenças nos valores de reflectância das imagens não possuem um significado para a classificação desejada, sendo mais fácil a detecção pela interpretação visual do que por métodos automatizados. Como exemplo, uma área de cultivo pode apresentar diferentes estágios da cultura que são retratados na imagem com diferentes espectros, desde o preparo para o plantio, quando o solo é totalmente exposto, até os mais avançados estádios da cultura, com grande recobrimento do solo pela folhagem.
As mudanças que foram extraídas pela série temporal de imagens são utilizadas para avaliar a dinâmica espacial e quantificar o aumento das áreas de cultivo no município de LEM. Na interpretação visual as áreas com pivô central foram classificadas detalhadamente devido à sua importância tanto no aspecto econômico, que indica o investimento local, como também pelo seu aspecto ambiental, devido à utilização dos recursos hídricos.

\section{RESULTADOS E DISCUSSÕES}

\subsection{Relação do crescimento da área de agropecuária com a política agrícola}

A classificação por interpretação visual da imagem PRISM/ALOS do município de LEM para 2008

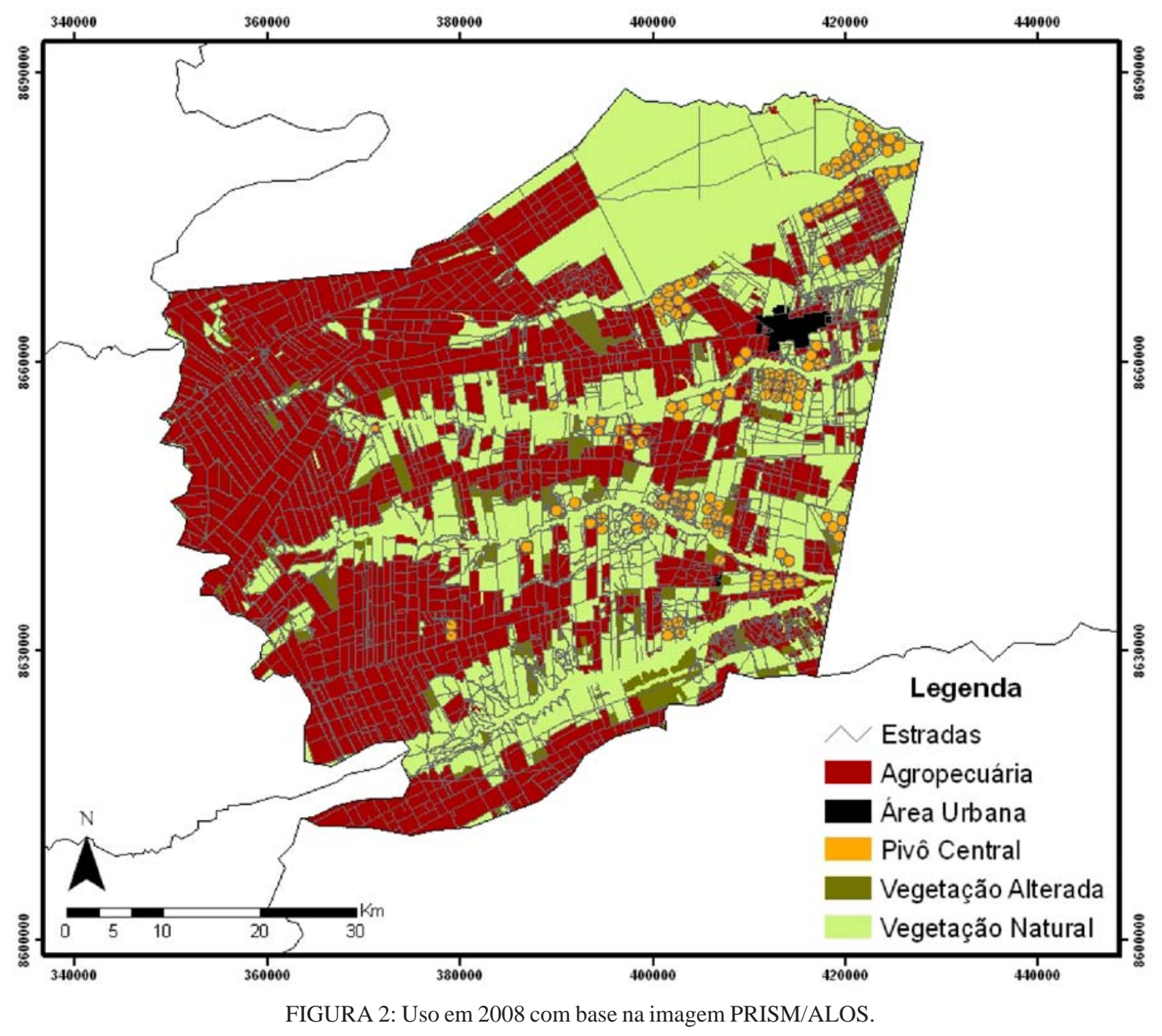

Sociedade \& Natureza, Uberlândia, 21 (3): 315-326, dez. 2009 
Análise das mudanças do uso agrícola da terra a partir de dados de sensoriamento remoto multitemporal no Município de Luis Eduardo Magalhães (BA - Brasil)

Aline Brignol Menke, Osmar Abílio de Carvalho Junior, Roberto Arnaldo Trancoso Gomes, Éder de Souza Martins, Sandro Nunes de Oliveira

é apresentada na FIG. 2. Observa-se que para esta data tem-se $42,75 \%$ do município ocupado pela vegetação nativa (cerrado), 49,38\% ocupado pela atividade agropecuária e 0,55\% pela área urbana.

A dinâmica da evolução do uso da Terra pode ser observada na FIG. 3 que apresenta uma série tem- poral proveniente da classificação das imagens Landsat. Observa-se a intensa taxa de mudança e conversão do cerrado para áreas de agropecuária. Entre 1987 a 2008, a vegetação nativa de cerrado teve uma diminuição devido à expansão da agropecuária, cuja área teve uma expansão de aproximadamente $50 \%$.

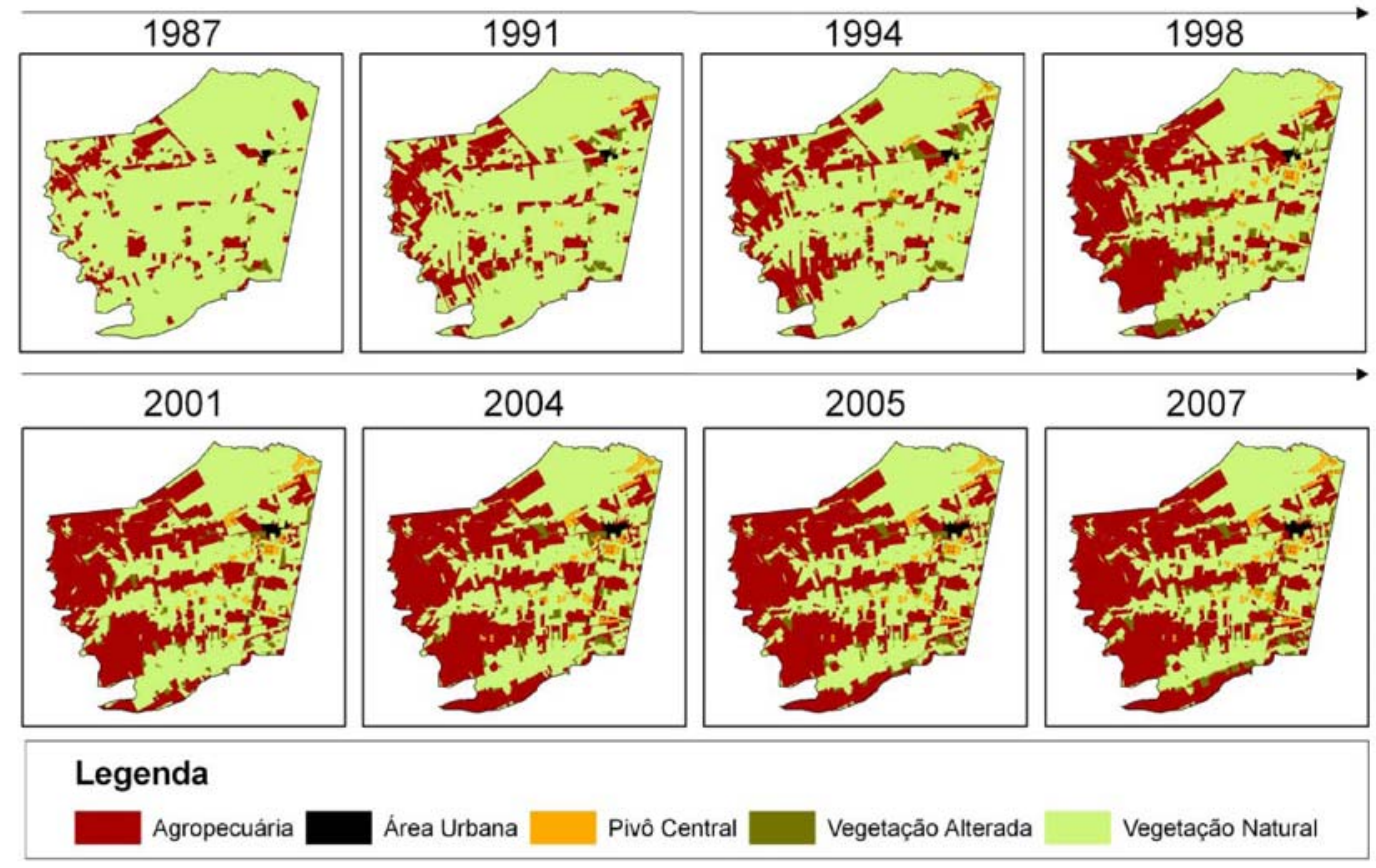

FIGURA 3: Evolução temporal do uso no município de Luis Eduardo Magalhães, localizada no oeste do Estado da Bahia. a) uso em 1987, b) uso em 1991, c) uso em 1994, d) uso em 1998, e) uso em 2001, f) uso em 2004, g) uso em 2005, e h) uso em 2007.

Durante este período houve uma intensa transformação do setor agrícola, devido às reformas e mudanças institucionais da economia brasileira provenientes dos planos de estabilização, contingenciamento dos gastos públicos, privatizações e a abertura comercial e financeira da economia.

Entre 1985 a 1994 o governo concentrou esforços na busca da estabilização financeira. Para tanto, a partir de 1991 efetua uma diminuição dos subsídios estatais e aboliu a política de preços mínimos, ocasionando uma reestruturação do sistema produtivo agrário. Assim, o governo diminui a sua participação na comercialização agrícola, tanto na compra via preços mínimos remuneradores como da venda de estoques agrícolas, substituindo-os pelo financiamento da estocagem (EGF) e pela adoção de novas regras de comercialização (REZENDE, 2003). Nesta conjuntura, o financiamento dos agricultores passa a ter uma grande contribuição do sistema privado e agroindustrial, que substitui em parte o Estado Federal (BELIK \& PAULLILO, 2001; GASQUES \& VILLA-VERDE, 1996; LEITE, 2000). As políticas de financiamento e comercialização das safras tornam-se mais independentes do intervencionismo e da coordenação do sistema público definindo novas relações entre as iniciativas públicas e privadas mais integradas ao agronegócio (BARBOSA, 2008).

Esta transferência de parte do financiamento agrícola ao sistema de mercado favoreceu o desenvolvimento de um crédito privado, como o "Contrato de Compra e Venda de Soja Verde” (BARBOSA, 2008). Este contrato mercantil de entrega futura com preços 
fixos ou a fixar possui base legal no código comercial e civil em seus artigos 192 e 1126, respectivamente, que tratam dos contratos futuros e dos registros (GASQUES \& VERDE, 1996). Assim, o preço-base é calculado tomando por referência o preço do mercado futuro, e os contratos são registrados em cartório. Nesta operação a fonte do dinheiro é proveniente de "tradings" (exportadores e indústrias processadoras de grãos que detêm as principais marcas de fertilizantes), que financiam os produtores na aquisição dos insumos. Estas novas fontes de recursos privados se instalam e estruturam grande parte da cadeia produtiva instalada no Município de LEM, que se mantêm até os dias de hoje.

Na segunda metade da década de 90, com a estabilização do Plano Real (lançado no final de 1993) ocorre a diminuição nos preços das terras e dos produtos agrícolas, efeitos atribuídos ao aumento das taxas de juros e apreciação cambial. A redução do preço das máquinas e insumos provocou um aumento no Brasil em 50\% do consumo de fertilizantes entre 1992 e 1997 (HELFAND e REZENDE, 2003). Neste cenário é mantida uma forte expansão da agricultura intensiva no município de LEM (FIG. 3), alimentada por mais máquinas, fertilizantes e outros insumos. Em consonância com o crescimento nacional o município em estudo apresenta um intenso aumento da área agropecuária.

Deve-se também salientar a participação estadual do governo da Bahia a partir do ano 2000, com a implantação de programas para alavancar a produção do Estado, com repercussões diretas na região do Oeste da Bahia: (a) Programa de Investimento para Modernização da Agricultura Baiana (AGRINVEST); (b) Programa de Desenvolvimento da Cafeicultura do Oeste do Estado da Bahia (PRODECAF); (c) Programa de Incentivo ao Algodão na Região Oeste do Estado da Bahia (PROALBA); e (d) Programa de Promoção do Desenvolvimento da Bahia (PROBAHIA). O AGRINVEST começa no ano 2000, promovendo a equalização de $50 \%$ dos encargos financeiros, durante o período de carência, com caráter modernizante, limitados a 6\% ao ano (SEAGRI-BA, 2003b). No mesmo ano inicia o PRODECAF com objetivo de desenvolver ações integradas de assistência técnica, gerencial e de crédito, buscando aumentar a produção, a expan- são da área cultivada e a elevação de ganhos de produtividade. Em 2001, institui o PROALBA com objetivo de apoiar os empreendimentos ligados à cadeia do agronegócio do algodão. Este programa concede a redução de até 50\% ICMS incidente sobre o valor da comercialização do algodão em pluma desde que o produtor obedeça a critérios de manejo da lavoura e qualidade da produção, pré-estabelecidos. Deste incentivo 10\% são destinados para o Fundo para o Desenvolvimento do Agronegócio do Algodão (FUNDEAGRO), que objetiva dar suporte financeiro aos projetos de pesquisa, defesa sanitária, monitoramento ambiental e promoção do agronegócio do algodão. Para usufruir do benefício fiscal do PROALBA, o produtor contribuirá com o valor correspondente a 10\% do imposto devido (SEAGRI-BA, 2003).

Um importante marco para agricultura foi à desvalorização do Real em 1999, que favoreceu o agronegócio protegendo da concorrência externa e aumentando o grau de competitividade do setor agrícola no mercado internacional (MAIA et al., 2005). No entanto, no início este estímulo foi parcialmente anulado pela queda dos preços internacionais, que voltam a subir a partir de 2002 provocando um grande dinamismo na agricultura brasileira (BRANDÃO et al., 2006). Em 2003 e 2004, o preço da saca de soja atingiu picos da elevação no mercado que provocou as quebras contratuais de venda antecipada com as indústrias e/ ou “tradings" (REZENDE, 2008). O município de LEM criado em 2001 apresenta um significativo acréscimo do valor da produção nos anos de 2003, 2004 e 2005 devido ao aumento do preço da soja e do algodão no mercado internacional (CEPEA, 2006; IBGE, 2007) (TAB. 1).

Nos anos de 2005 e 2006 observa-se no Brasil uma diminuição de contratos como as "tradings" devido aos seguintes fatores (REZENDE, 2008): (a) preço baixo da saca de soja, devido ao excesso de oferta; (b) expectativa do produtor de elevação de preços durante a safra, como aconteceu em 2003 e 2004; e (c) ocorrência de quebra ou descumprimento de contrato nas duas últimas safras, que fez com que fossem exigidas maiores garantias para obtenção de crédito. Estes fatos fizeram com que houvesse uma retração no crescimento das áreas de cultivo no município em estudo.

Desta forma, durante toda a década de 90 e 
no início dos anos 2000 observa-se no município de LEM um grande aumento da área cultivada (FIG. 4a) e da produção agropecuária. A partir de 2003 observase uma menor incorporação de novas terras, apesar do valor da produção no município apresentar tendência de aumento, provavelmente devido ao aprimoramento tecnológico (TAB. 1). Apesar da taxa de crescimento diminuir, em 2005 verifica-se que a área da agrope- cuária no município suplanta a área de vegetação nativa (FIG. 4b). Em parte a diminuição da conversão é devida ao esgotamento de terras para agropecuária, uma vez que uma porção da área deve ser destinada por lei para reserva legal e preservação permanente. Desta forma, diminui a oferta de terras que passam a ter uma acentuada valorização para novos empreendimentos.

a)

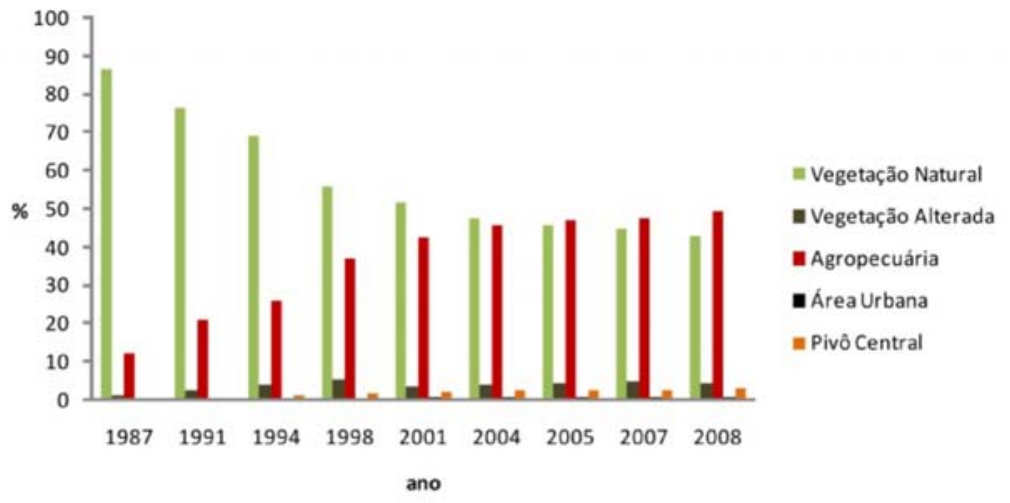

b)

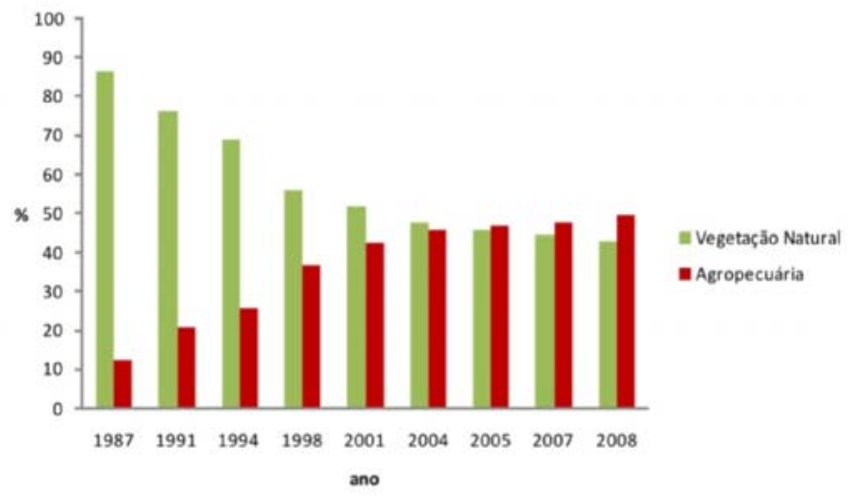

c)

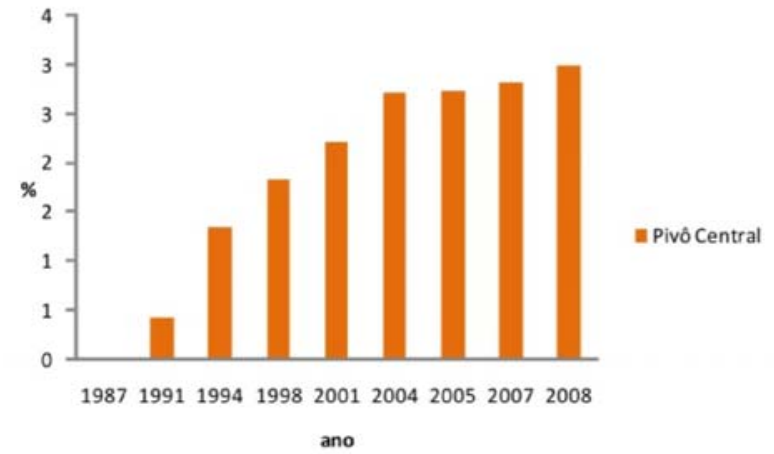

FIGURA 4: Gráficos da análise multitemporal dos usos em Luis Eduardo Magalhães. a) Percentual de área de uso em Luis Eduardo por classes, nos anos em estudo; b) percentual de área em uso classes vegetação natural e agropecuária, nos anos em estudo, c) crescimento da área de pivô central, nos anos em estudo. 

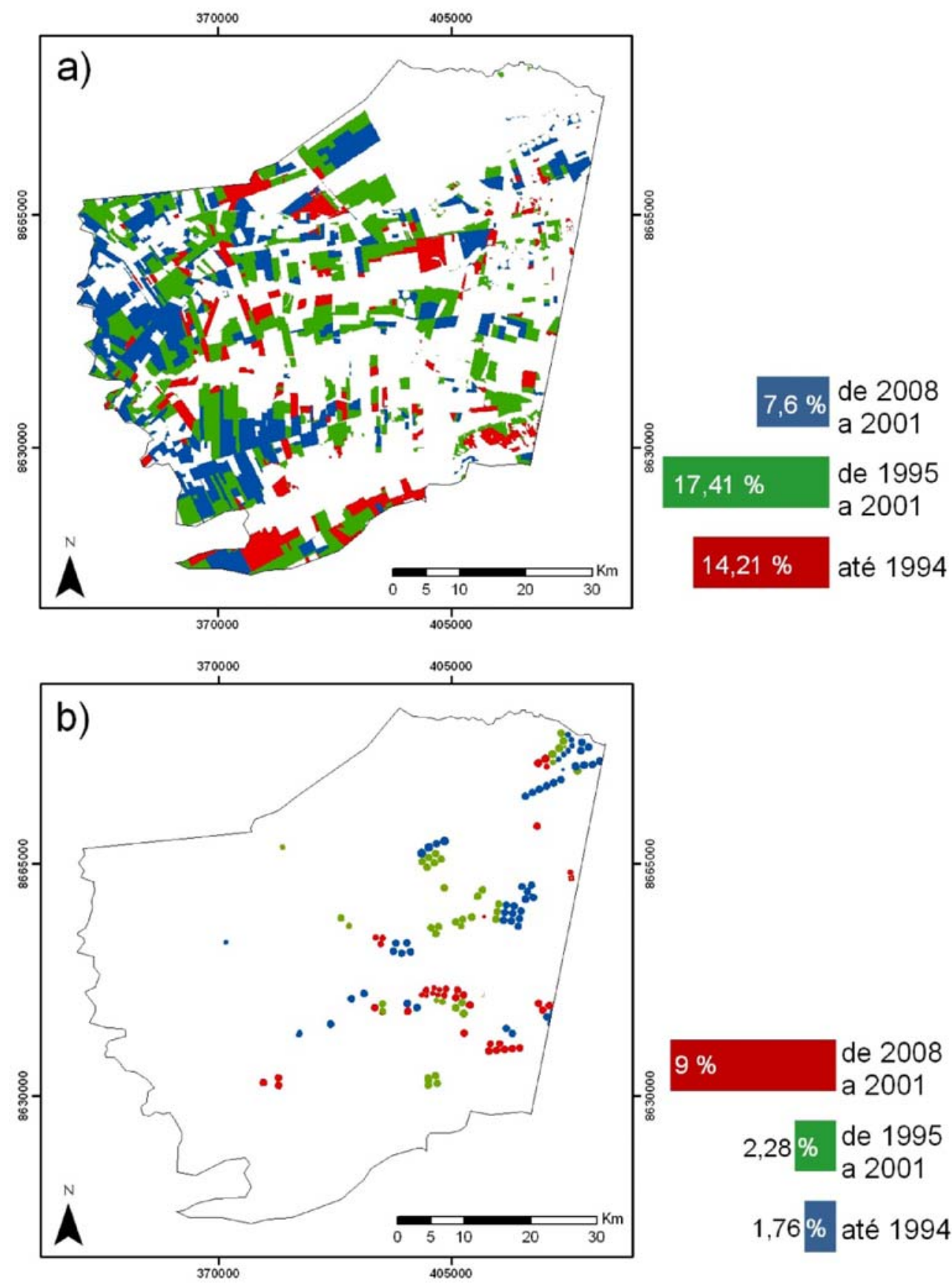

$14,21 \%$ até 1994

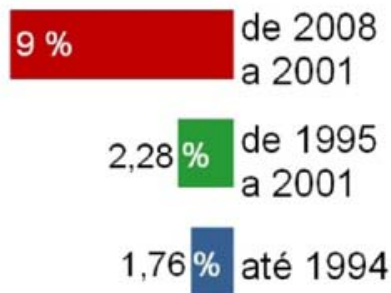

FIGURA 5. (a) crescimento percentual da área de agropecuária em relação à área total, para o intervalo de anos em estudo.

(b) crescimento percentual da área de pivôs em relação à área total, para o intervalo de anos em estudo.

Cabe salientar que as parcelas identificadas como atividade agrícola podem não estar, necessariamente, com culturas. Eventualmente, as áreas que foram cultivadas em um ano podem entrar em descanso no ano seguinte, sendo computadas como área de uso da atividade agropecuária.

\subsection{Padrões e Vetores do Crescimento Agropecuário}

Outro importante ponto para discussão é o padrão espacial da expansão agrícola dentro do município. A expansão ocorre principalmente ao longo das linhas de cumeeira do relevo, onde estão localizadas 
as estradas principais para o escoamento da produção (FIG. 5). A porção oeste é a mais ocupada devido à maior precipitação que favorece o plantio de sequeiro. Esta expansão ocorre principalmente na década de 90.

No entanto, as áreas com pivô central que demonstram o maior aprimoramento e incremento tecnológico expandiram-se na década atual (FIG. 5b).
Em 1987 não havia nenhum pivô central no município, já em 2008 foram identificados 126 pivôs, o que corresponde a aproximadamente 9\% da área total (FIG. 4c). Observa-se que a distribuição das áreas de pivô central está localizada na porção leste do município, onde a pluviosidade é menor e os rios apresentam maior vazão, favorecendo a irrigação.

\begin{tabular}{llll}
\hline Ano & Área plantada (ha) & $\begin{array}{l}\text { Quantidade } \\
\text { Produzida (t) }\end{array}$ & $\begin{array}{l}\text { Valor da produção } \\
(\mathbf{1} \mathbf{0 0 0} \mathbf{R} \$)\end{array}$ \\
\hline $\mathbf{2 0 0 1}$ & 206025 & 545040 & 169581 \\
$\mathbf{2 0 0 2}$ & 212733 & 515163 & 227069 \\
$\mathbf{2 0 0 3}$ & 181809 & 457328 & 273706 \\
$\mathbf{2 0 0 4}$ & 171962 & 617856 & 390171 \\
$\mathbf{2 0 0 5}$ & 173331 & 584165 & 309755 \\
$\mathbf{2 0 0 6}$ & 176521 & 491609 & 255660 \\
$\mathbf{2 0 0 7}$ & 178134 & 526837 & 318587 \\
\hline
\end{tabular}

TABELA 1: Tabela de área plantada, quantidade produzida e valor da produção em Luis Eduardo Magalhães entre 2001 e 2007.

Um fator preocupante é o padrão de crescimento homogêneo e contínuo das áreas de plantio, onde não se observa a manutenção de cerrado nativo. Desta forma, este crescimento demonstra uma falta de planejamento ambiental formando extensas áreas com uso agrícola. As áreas mais preservadas consistem nas porções mais próximas dos canais fluviais onde o entalhamento do talvegue gera, por erosão diferencial, uma ruptura do relevo que desfavorece a mecanização. Outra extensa área que ainda se mantêm preservada ocorre na porção nordeste do município. Por ser a última grande área contínua de vegetação nativa devese estimular a sua preservação por parte do governo municipal.

\section{CONCLUSÃO}

A análise multitemporal utilizando o método de pós-classificação mostrou-se bastante eficiente na descrição da evolução do uso da Terra. Os polígonos gerados poderão ser retrabalhados posteriormente na atualização e monitoramento do uso da Terra no município. Observou-se um acentuado crescimento da agricultura na década de 90, com uma retração do avanço a partir de 2003. No entanto o aumento nesta década das áreas de irrigação por pivô central demonstra que a produção no município de LEM vem se capitalizando e aperfeiçoando nos últimos anos. Atualmente, o município ocupa um papel de importância na economia baiana, com a incorporação de produtos de grande valor no mercado internacional como a soja e o algodão. Todavia, faz-se necessário um programa de monitoramento no município visto que o ritmo de crescimento pode comprometer os recursos naturais locais.

\section{REFERÊNCIAS}

BARBOSA, G.J.; COUTO, E.P. Evolução das políticas agrícolas e o incentivo à iniciativa privada na agricultura brasileira. In: Congresso da Sociedade Brasileira de Economia, Administração e Sociologia Rural. 46., 2008, Rio Branco-AC. Anais... 2008. 20 p. Disponível em: <http://www.sober.org.br/palestra/9/896.pdf>. Acesso em: 10 jul. 2009.

BELIK, W.; PAULILLO, L. F. O financiamento da 
produção agrícola brasileira na década de 90: ajustamento e seletividade. In: LEITE, S (Ed.). Políticas públicas e agricultura no Brasil. Porto Alegre: Editora UFRGS, 2001, p. 95-120.

BORLAUG, N.E. Feeding a world of 10 billion people: the miracle ahead. In: Bailey, R. (Ed.). Global warming and other eco-myths. Competitive Enterprise Institute, Roseville, EUA, p. 29-60, 2002.

BRANDÃO, A.S.P.; REZENDE, G.C. \& MARQUES, R.W.C. Crescimento agrícola no período 1999/2004: a explosão da soja e da pecuária bovina e seu impacto sobre o meio ambiente. Economia Aplicada, São Paulo, v.10, n.2, p. 249-266, 2006.

CENTRO DE ESTUDOS AVANÇADOS EM ECONOMIA APLICADA - CEPEA. Indicadores de preços, 2006. Disponível em: www.cepea.esalq.usp.br. Acesso em: 01 de fev. 2009.

COPPIN, P.; JONCKHEERE, I.; NACKAERTS, K.; MUYS, B.; LAMBIN, E. Review article digital change detection methods in ecosystem monitoring: a review. International Journal of Remote Sensing, v. 25, n. 9, p. 1565-1596, 2004.

CUNHA, T.J.F.; MACEDO, J.R.; RIBEIRO, L.P.; PALMIERI, F.; FREITAS, P.L. \& AGUIAR, A.C. Impacto do manejo convencional sobre propriedades físicas e substâncias húmicas de solos sob Cerrado. Ciência Rural, Santa Maria, v.1, n.1, p. 27-36, 2001.

DE BRUIN, S. Querying probabilistic land cover data using fuzzy set theory. International Journal of Geographical Information Sciences, v.14, p.359-372, 2000.

FONSECA, M.G.D.; SILVEIRA, J.M.F.J. da \& DAL POZ, M.E. Biotecnologia Vegetal e Produtos Afins. In: SILVEIRA, J.M.F.J da, DAL POZ, M.E e ASSAD, A. (Eds.) Biotecnologia e Recursos Genéticos: Desafios e Oportunidades para o Brasil. Campinas: FINEP/ Instituto de Economia da Unicamp, $1^{\text {a }}$ ed, 2004.

GASPAR, M.T.P. Sistema Aqüífero Urucuia: Caracte- rização regional e proposta de gestão. 2006. 158 f. Tese (Doutorado em Geologia) - Instituto de Geociências - Universidade de Brasília, Brasília.

GASQUES, J. G.; VILLA VERDE, C. M. Novas fontes de recursos, propostas e experiências de financiamento rural. Revista de Economia e Sociologia Rural. v.34, n.3 e 4, p. 39- 80, 1996.

HELFAND, S.M.; REZENDE, G.C. Região e espaço no desenvolvimento agrícola brasileiro. Rio de Janeiro: IPEA, 2003. 390p.

HOWARTH, P.J. \& MICKWARE, G.M. Procedures for change detection using Landsat digital data. International of Remote Sensing, v. 2, p. 277-279, 1981.

IGARASHI, T. ALOS mission requirement and sensor specifications. Advances in Space Research, v.28, n.1, p. 127-131, 2001.

INSTITUTO BRASILEIRO DE GEOGRAFIA E ESTATÍSTICA - IBGE. Atlas Nacional do Brasil, 4a edição. 2002.

INSTITUTO BRASILEIRO DE GEOGRAFIA E ESTATÍSTICA - IBGE. Produção Agrícola Municipal: cereais, leguminosas e oleaginosas. Diretoria de Pesquisas, Coordenação de Agropecuária, 2007.

INSTITUTO INTERAMERICANO DE COOPERAÇÃO PARA A AGRICULTURA - IICA. Informe nacional da situação e das perspectivas da agricultura/ 2007: Brasil, 2007. Disponível em: <http://www. iica.org.br/Docs/Noticias/IICAdivulgaDocumentos RefletemAgriculturaBrasil_InformeNacionalBrasil 2007.pdf>. Acesso em: 03 de mar. 2009.

JAXA. 2006. ALOS Product Format Description. Disponível em: <http://stage.tksc.jaxa.jp/eorcalos/ PRISM_L1_J_ENa.zip> Acesso em 7 abril 2007.

KOCAMAN, S.; GRUEN, A., 2008. Orientation and Self-calibration of ALOS PRISM Imagery. The Photogrammetric Record, v.23, n.123, p. 323-340. 
Análise das mudanças do uso agrícola da terra a partir de dados de sensoriamento remoto multitemporal no Município de Luis Eduardo Magalhães (BA - Brasil)

Aline Brignol Menke, Osmar Abílio de Carvalho Junior, Roberto Arnaldo Trancoso Gomes, Éder de Souza Martins, Sandro Nunes de Oliveira

LEITE, C.A.M. Política agrícola para o setor rural em transição. In: SANTOS, M.L.; VIERIA, W.C. (Eds.). Agricultura na virada do milênio: velhos e novos desafios. Viçosa: Suprema, 2000. p. 193-216.

MAIA, A.G.; DEDECCA, C.S.; VIEIRA FILHO, J.E.R.; SILVEIRA J.M.A. Evolução Recente da Ocupação e do Rendimento no Setor Agrícola In: Congresso da Sociedade Brasileira de Economia e Sociologia Rural, 43. Ribeirão Preto. Anais... Ribeirão Preto: SOBER, 2005.

MAS, J. F. Monitoring land-cover changes: a comparison of change detection techniques. International Journal of Remote Sensing, n. 18, p. 711725, 1999.

MUNYATI, C. Wetland change detection on the Kafue Flats, Zambia, by classification of a multitemporal remote sensing image dataset. International Journal of Remote Sensing, v.21, n.9, p. 1787-1806, 2000.

NARUMALANI, S.; MISHRA, D.R.; ROTHWELL, R.G. Change detection and landscape metrics for inferring anthropogenic processes in the greater EFMO area. Remote Sensing of Environment, v.91, p.478489, 2004.

REZENDE, G.C. Estado, macroeconomia e agricultura no Brasil. Porto Alegre: Editora da UFRGS/ IPEA, 2003.

REZENDE, C.L. Pacta sunt servanda? Quebra dos contratos de soja verde. 2008. 144p. Tese (Doutorado em Administração) - Faculdade de Economia, Administração e Contabilidade (FEA), Universidade de São Paulo, São Paulo.

ROCHA, M.B.B.; ROSA, R. Caracterização do meio físico e monitoramento do uso da terra em 1985 e 2005 do município de Araxá - MG. Caminhos da Geografia (UFU. Online), v. 9, p. 95-107, 2008.

SANO, E. E.; ROSA, R.; BRITO, J.L.S.; FERREIRA, Laerte Guimarães. Mapeamento semidetalhado do uso da terra do Bioma Cerrado. Pesquisa Agropecuária
Brasileira (Online), v. 43, p. 153-156, 2008.

SANO, E.E.; ROSA, R.; BRITO, J.L.S.; FERREIRA, L.G. Land cover mapping of the tropical savanna region in Brazil. Environmental Monitoring and Assessment, v. 1, p. 1-12, 2009.

SECRETARIA DE AGRICULTURA, IRRIGAÇÃO E REFORMAAGRÁRIA DO ESTADO DA BAHIASEAGRI-BA. Apoio a produtores do sudoeste e do Médio São Francisco teve o investimento dobrado. 12 ago. 2003. Disponível em: <www.seagri.ba.gov.br> . Acesso em: 20 de março de 2009.

SICSÚ, A.B.; LIMA, J.P.R. Fronteiras agrícolas no Brasil: a lógica de sua ocupação recente. Nova Economia, Belo Horizonte, v.10, n.1, p. 109-138, 2000.

SINGH, A. Digital change detection techniques using remotely-sensed data. International Journal of Remote Sensing, v.10, p. 89-1003, 1989.

SUPERINTENDÊNCIA DE ESTUDOS ECONÔMICOS E SOCIAIS DA BAHIA - SEI. Panorama da migração dos municípios baianos em 1995-2000. Salvador: SEI, 2007.

TADONO T., SHIMADA M., WATANABE M., HASHIMOTO T.; IWATA T. Calibration and Validation of PRISM Onboard ALOS. In: ISPRS Congress, 20., Istanbul, Turkey. The International Archives of Photogrammetry, Remote Sensing and Spatial Information Sciences, v.35, part. B1, 2004. p. 13-18.

WESER, T.; ROTTENSTEINER, F.; WILLNEFF, J. \& FRASER. C.S. An improved pushbroom scanner model for precise georeferencing of ALOS prism imagery. In: ISPRS Congress, 21., Beijing, China. The International Archives of the Photogrammetry, Remote Sensing and Spatial Information Sciences. v. 37. Part. B1, 2008. p. 724-729. 